\title{
PHONON-ASSISTED KINETICS OF ELECTRON-HOLE PAIR IN TWO-BAND MODEL
}

\author{
E. Klotins \\ Institute of Solid State Physics, University of Latvia, Kengaraga 8, LV-1063 Riga, Latvia \\ E-mail: klotins@cfi.lu.lv
}

Received 5 May 2014; revised 10 June 2014; accepted 23 September 2014

\begin{abstract}
Photoexcited kinetics of electrons and holes in two-band dielectric including phonon-assisted generation of the electron-hole pair is modelled by combining the quantum field and solid-state band theory. These methods create an explicit time-domain representation of photoinduced processes without resorting to the perturbation theory for the electromagnetic field. Input entities for the two-band model are dispersion relations for the electron and hole, the band-gap, and the phonon frequencies computed by nonrelativistic methods or found experimentally. The phonon-assisted electron-hole pair kinetics is initiated by the BakerCampbell-Hausdorff canonical transformation accounting for the back reaction of phonons to the electron-hole subsystem. The output is a unifying phonon-assisted description of the distribution function of electron and hole quasiparticles.
\end{abstract}

Keywords: two-band model, electron-hole excitation, Baker-Campbell-Hausdorf canonical transformation

PACS: 71.15.Rf, 63.20.kd, 42.50.Ct

\section{Introduction}

The electron-hole (e-h) pairs are structures with a unique combination of solid-state and atom-like properties. The atomic view emphasizes electron and hole properties at short distances obeying relativistic conditions and formalism of the quantum field theory (QFT) according to which the concept of a single point-like particle breaks down completely [1]. The solid-state view emphasizes particle properties of electrons and holes.

The impact of phonons is a long standing problem distinguished by the type of particle-phonon interaction elaborated in pioneering models of Fröhlich [2, 3] and Holstein [4, 5] for optical phonons with a polar long-range interaction and non-polar short-range interaction, correspondingly. In modern approaches [ [6] the electromagnetic radiation and electron-phonon interaction are central and play a crucial role in modelling polymers, ferroelectrics, and several important classes of perovskites. Contemporary techniques to describe phonon-assisted photoexcited dynamics of electrons and holes range from $a b$ initio time-dependent density functional theory [⿰亻] and non-adiabatic molecular dynamics [8] to the advanced semiconductor (SC) approach [9]. Nevertheless, restrictions of the SC approach appear in a strong field and non- adiabatic effects that cannot be eliminated within the existing rotating wave and perturbation approaches. Although the SC approach is a quantum field approach, it has a perspective for joint application with the quantum electrodynamics (QED) considered in this work.

On the other hand, quantum physics of classical particles in the relativistic limit of the QFT is a fundamentally new approach to solid state [1]. The simplest result one can expect from the QFT is the birth and annihilation of non-interacting particles. It is definitely the problem of the phonon-assisted kinetics of e-h pairs.

In this work we are employing a rich experience of the QFT and QED in describing the nonperturbative kinetics of e-h excitations [10], strong field generalization of the interband transitions [11], interband tunnelling [12], and also using some concepts of solid-state band theory [13]. The QED approach [10-12] starts with the dispersion relations for electron and hole, and band-gap value as input parameters are computed by nonrelativistic methods or found experimentally. Levels of description range from a direct band-gap, effect of electromagnetic radiation, and the phonon-assisted e-h interaction.

A mathematical tool used here to describe e-h pairs, without resorting to the perturbation theory for 
the electromagnetic field, is a complex scalar KleinGordon (KG) type oscillator equation for a joint KG field function for the non-interacting electron and hole [11]. The solution of the oscillator equation obeys the general dispersion law and formally is given by integrals over the 4-dimensional space, second quantized Hamiltonian, canonical momentum operators, and finally by the distribution function for electrons and holes.

This paper concentrates on some elements of generalization of the known case [11] where the electron and hole remain non-interacting unless electromagnetic field is switched on. In the other case the interaction with the phonon subsystem is based on the electron-phonon interaction Hamiltonian [13-15] structured by mixed electronic and phonon operators. We turn to the Baker-Campbell-Hausdorff $(\mathrm{BCH}) \mathrm{ca}-$ nonical transformation of the interaction Hamiltonian [16] that disconnects the electron-phonon and hole-phonon interaction to a purely electronic and hole problem, so capturing both the effect of strong field electromagnetic interaction and the back reaction of phonons. Our initial objective is not to make quantitative predictions for a specific experimental realization but to contribute to understanding the role phonons play in the kinetics of e-h excitation.

The arrangement of this work is the following: Section 2 considers the basic framework of a complex KG approach for a system defined by the e-h pair fixed to hypersurfaces in the energy-quasi-momentum space. Both direct and indirect conduction and valence bands are allowed with e-h states obeying arbitrary dispersion laws. Section 3 addresses the KG field functions derived in accord with the case specific dispersion laws. In Section 4, the amplitude of interband transitions and the e-h distribution function are shown to be the starting point of phonon-assisted e-h kinetics. In Section 5, the e-h phonon interaction is included making use of the $\mathrm{BCH}$ canonical transformation constituting the back reaction to the e-h subsystem. The last Section 6 contains a short summary of the work. Natural units, $\hbar=c=1$, are used.

\section{Basic framework}

The QFT framework we address combines ideas from special relativity and quantum mechanics. Application of this framework to the condensed matter physics is motivated by a real physical situation where the number of particles is not constant and keeps changing as a result of interaction with the lattice environment and external electromagnetic radiation. A route to relativistic quantum mechanics starts with then classical nonrelativistic particle theory. In case of a non-interacting electron and hole, their states are distinguished exclusively by potential energy and quasimomenta. The joint e-h description is obtained by the product of electron and hole states and their first quantization.

The system is defined by an e-h pair fixed to hypersurfaces in the energy-quasi-momentum space with e-h states in the conduction and valence bands [11]:

$$
E_{\mathrm{c}}=\frac{\Delta}{2}+\varepsilon_{\mathrm{c}}(\mathbf{p}), \quad E_{\mathrm{v}}=-\frac{\Delta}{2}-\varepsilon_{\mathrm{v}}(\mathbf{p}),
$$

where dispersion laws of the kinetic energy $\varepsilon_{c}(\mathbf{p})$ and $\varepsilon_{\mathrm{v}}(\mathbf{p})$ are either assumed on the phenomenological basis or calculated by methods of nonrelativistic quantum mechanics. Here, the band-gap $\Delta$ and the band boundaries represented by dispersion laws $\varepsilon_{c, v}(\mathbf{p})$ remain presumably invariable quantities. Electron and hole (below regarded as an antiparticle to the electron) states are correlated and allow joint description in terms of the general second order dispersion:

$$
\begin{aligned}
& \left(E-E_{\mathrm{c}}\right)\left(E-E_{\mathrm{v}}\right)= \\
& {\left[E-\frac{\Delta}{2}-\varepsilon_{\mathrm{c}}(\mathbf{p})\right]\left[E+\left(\frac{\Delta}{2}\right)+\varepsilon_{\mathrm{v}}(\mathbf{p})\right]=0 .}
\end{aligned}
$$

For quadratic dispersion and in effective mass approximation Eq. (2) becomes

$$
\begin{aligned}
& \left(E-E_{\mathrm{c}}\right)\left(E-E_{\mathrm{v}}\right)=\left[E-\frac{\Delta}{2}-\left(\frac{\mathbf{p}^{2}}{2 m_{\mathrm{e}}}\right)\right] \\
& \times\left[E+\left(\frac{\Delta}{2}\right)+\left(\frac{\mathbf{p}^{2}}{2 m_{\mathrm{h}}}\right)\right]=0 .
\end{aligned}
$$

In case of an indirect band-gap, the dispersion laws in Eq. (1) are written as $\varepsilon_{c}\left(\mathbf{p}-\mathbf{p}_{0 c}\right)$ and $\varepsilon_{v}\left(\mathbf{p}+\mathbf{p}_{0 v}\right)$, where the difference between the electron and hole momentum is $\left(\mathbf{p}_{0 \mathrm{c}}-\mathbf{p}_{0 \mathrm{v}}\right)$. The first quantized free-field equation of motion of Eq. (3) is given by relation

$$
\left[\hat{E}-\frac{\Delta}{2}-\varepsilon_{\mathrm{c}}(\hat{\mathbf{p}})\right]\left[\hat{E}+\frac{\Delta}{2}+\varepsilon_{\mathrm{v}}(\hat{\mathbf{p}})\right] \Psi(\mathbf{x}, t)=0,
$$

where $\Psi(x, t)$ is a total wave function and $\hat{E} \Rightarrow \mathrm{i} \hbar \frac{\partial}{\partial t}$. In this first quantization notation the kinetic Eq. (4) reads as follows:

$$
\begin{aligned}
& {\left[-\frac{\partial^{2}}{\partial t^{2}}+\left(\mathrm{i} \frac{\partial}{\partial t}\right) \varepsilon_{\mathrm{v}}(\hat{\mathbf{p}})-\left(\frac{\Delta}{2}\right)^{2}-\frac{\Delta}{2} \varepsilon_{\mathrm{v}}(\hat{\mathbf{p}})-\varepsilon_{\mathrm{c}}(\hat{\mathbf{p}})\right.} \\
& \left.\times\left(\mathrm{i} \frac{\partial}{\partial t}\right)-\varepsilon_{\mathrm{c}}(\hat{\mathbf{p}})\left(\frac{\Delta}{2}\right)-\mathcal{E}_{\mathrm{c}}(\hat{\mathbf{p}}) \varepsilon_{\mathrm{v}}(\hat{\mathbf{p}})\right] \Psi(\mathbf{x}, t)=0 .
\end{aligned}
$$


This first step toward relativistic description is similar to the deducing canonical KG equation for a free particle of the rest mass and 3-momentum. However, the difference is that the quantized kinetic equation for the e-h pair comprises extra dispersion specific terms managed in oscillator equation technique and reexamined in this Section. With application to the two-band model the transition from Eq. (1) to Eq. (5) is very important for our aim and was motivated earlier in [12].

For subsequent application of the Lagrange-Hamilton formalism the kinetic Eq. (5) is transformed to the uniform differential form of the second order with respect to time without the first order time derivative stipulated by the asymmetry of electron and hole energy states in the reference energy-quasi-momentum frame. Such equation is obtained if the auxiliary function $\Phi(\mathbf{x}, t)$ is introduced:

$$
\Psi(\mathbf{x}, t)=\exp \left\{\frac{\mathrm{i}}{2}\left[\varepsilon_{\mathrm{v}}(\hat{\mathbf{p}})-\varepsilon_{\mathrm{c}}(\hat{\mathbf{p}})\right] t\right\} \Phi(\mathbf{x}, t),
$$

which turns Eq. (5) into the oscillator equation:

$$
\begin{aligned}
& \ddot{\Phi}(\mathbf{x}, t)+\left(\frac{\Delta^{2}}{4}+\frac{\Delta \varepsilon_{\mathrm{c}}(\hat{\mathbf{p}})}{2}+\frac{\left(\varepsilon_{\mathrm{c}}(\hat{\mathbf{p}})\right)^{2}}{4}+\frac{\Delta \varepsilon_{\mathrm{v}}(\hat{\mathbf{p}})}{2}\right. \\
& \left.+\frac{\varepsilon_{\mathrm{c}}(\hat{\mathbf{p}}) \varepsilon_{\mathrm{v}}(\hat{\mathbf{p}})}{2}+\frac{\left(\varepsilon_{\mathrm{v}}(\hat{\mathbf{p}})\right)^{2}}{4}\right) \Phi(\mathbf{x}, t)=0 .
\end{aligned}
$$

The terms at auxiliary function are assigned to the effective frequency and constitute the oscillator-type equation [17]:

$$
\ddot{\Phi}(\mathbf{x}, t)+\Omega^{2}(\hat{\mathbf{p}}) \Phi(\mathbf{x}, t)=0,
$$

with the effective frequency operator

$$
\Omega=\frac{1}{2}\left(\Delta+\varepsilon_{\mathrm{v}}(\hat{\mathbf{p}})+\varepsilon_{\mathrm{c}}(\hat{\mathbf{p}})\right) .
$$

The oscillator equation technique allows transforming the quantized kinetic equation in a uniform differential form for the auxiliary function applicable to the Lagrange-Hamilton formalism. The oscillator equation is restricted to the second order because of the mass-shell condition. The underlying physics is that particle creation is harmonized with the general dispersion law. In strong sense, the second order in the time oscillator (KG) equation is intermediate between the Schrödinger and Dirac equations. The level of approximation of the KG approach is the complex fields obeying relativistic requirements for e-h pairs with a zero spin. Development toward spin- $1 / 2$ particles within the framework of the Dirac equation is beyond the scope of this work.
The Lagrangian density for the auxiliary function depends on its second order time and space derivatives and the effective frequency operator comprising the parameters of band structure:

$$
\mathcal{L}(\mathbf{x}, t)=\frac{\partial}{\partial t} \Phi(\mathbf{x}, t) \frac{\partial}{\partial t} \Phi^{*}(\mathbf{x}, t)-\Omega^{2}(\hat{\mathbf{p}}) \Phi(\mathbf{x}, t) \Phi^{*}(\mathbf{x}, t) .
$$

The Lagrangian density for the total field function $\Psi(x, t)$ is found by inverse transformations:

$$
\begin{aligned}
& \Phi(\mathbf{x}, t)=\exp \left[-\frac{\mathrm{i} t\left(\varepsilon_{\mathrm{v}}(\hat{\mathbf{p}})-\varepsilon_{\mathrm{c}}(\hat{\mathbf{p}})\right)}{2}\right] \Psi(\mathbf{x}, t), \\
& \Phi^{*}(\mathbf{x}, t)=\exp \left[\frac{\mathrm{i} t\left(\varepsilon_{\mathrm{v}}(\hat{\mathbf{p}})-\varepsilon_{\mathrm{c}}(\hat{\mathbf{p}})\right)}{2}\right] \Psi^{*}(\mathbf{x}, t),
\end{aligned}
$$

where the term-by-term transformation of the Lagrangian density, Eq. (10), in the field function representation reads as

$$
\begin{aligned}
& \dot{\Phi}^{*}(\mathbf{x}, t) \dot{\Phi}(\mathbf{x}, t) \Rightarrow\left[-\frac{\mathrm{i}\left(\varepsilon_{\mathrm{v}}(\hat{\mathbf{p}})-\varepsilon_{\mathrm{v}}(\hat{\mathbf{p}})\right)}{2} \Psi(\mathbf{x}, t)+\dot{\Psi}(\mathbf{x}, t)\right] \\
& \times\left[+\frac{\mathrm{i}\left(\varepsilon_{\mathrm{v}}(\hat{\mathbf{p}})-\varepsilon_{\mathrm{c}}(\hat{\mathbf{p}})\right)}{2} \Psi^{*}(\mathbf{x}, t)+\dot{\Psi}^{*}(\mathbf{x}, t)\right] .
\end{aligned}
$$

As a result, the Lagrangian density in the field function representation is given by

$$
\begin{aligned}
& \mathcal{L}(\mathbf{x}, t)=\left(\left|\frac{\mathrm{i}\left(\varepsilon_{\mathrm{v}}(\hat{\mathbf{p}})-\varepsilon_{\mathrm{c}}(\hat{\mathbf{p}})\right)}{2} \Psi^{*}(\mathbf{x}, t)+\dot{\Psi}^{*}(\mathbf{x}, t)\right|^{2}\right. \\
& \left.+\Omega^{2}(\hat{\mathbf{p}})|\Psi|^{2}\right) .
\end{aligned}
$$

Expansion of the Lagrangian density,

$$
\begin{aligned}
& \mathcal{L}\left(\Psi, \Psi^{*} ; \dot{\Psi}, \dot{\Phi}^{*}\right)=\left\{\left[\frac{\left(\varepsilon_{\mathrm{c}}(\hat{\mathbf{p}})-\varepsilon_{\mathrm{v}}(\hat{\mathbf{p}})\right)^{2}}{4} \Psi \Psi^{*}\right.\right. \\
& +\frac{\mathrm{i}\left(\varepsilon_{\mathrm{c}}(\hat{\mathbf{p}})-\varepsilon_{\mathrm{v}}(\hat{\mathbf{p}})\right)}{2} \Psi \dot{\Psi}^{*}-\frac{\mathrm{i}\left(\varepsilon_{\mathrm{c}}(\hat{\mathbf{p}})-\varepsilon_{\mathrm{v}}(\hat{\mathbf{p}})\right)}{2} \Psi^{*} \dot{\Psi} \\
& \left.\left.+\dot{\Psi} \dot{\Psi}^{*}\right]-\Omega^{2}(\hat{\mathbf{p}}) \Psi^{*} \Psi\right\},
\end{aligned}
$$

gives the canonical moments and the Hamiltonian density as follows:

$$
\begin{aligned}
& \pi=\frac{\partial \mathcal{L}}{\partial \dot{\Psi}}=-\frac{\mathrm{i}\left(\varepsilon_{\mathrm{c}}(\hat{\mathbf{p}})-\varepsilon_{\mathrm{v}}(\hat{\mathbf{p}})\right)}{2} \Psi^{*}(\mathbf{x}, t)+\dot{\Psi}^{*}(\mathbf{x}, t), \\
& \pi^{*}=\frac{\partial \mathcal{L}}{\partial \dot{\Psi}^{*}}=+\frac{\mathrm{i}\left(\varepsilon_{\mathrm{c}}(\hat{\mathbf{p}})-\mathcal{E}_{\mathrm{v}}(\hat{\mathbf{p}})\right)}{2} \Psi(\mathbf{x}, t)+\dot{\Psi}(\mathbf{x}, t),
\end{aligned}
$$




$$
\begin{aligned}
& H=\pi \dot{\Psi}+\pi^{*} \dot{\Psi}^{*}-\mathcal{L}= \\
& -\frac{\left(\varepsilon_{\mathrm{c}}(\hat{\mathbf{p}})-\varepsilon_{\mathrm{v}}(\hat{\mathbf{p}})\right)^{2} \Psi(\mathbf{x}, t) \Psi^{*}(\mathbf{x}, t)}{4} \\
& +\Omega^{2}(\hat{\mathbf{p}}) \Psi(\mathbf{x}, t) \Psi^{*}(\mathbf{x}, t)+\dot{\Psi}(\mathbf{x}, t) \dot{\Psi}^{*}(\mathbf{x}, t) .
\end{aligned}
$$

Equations (14), (15), and (16) complete the freefield Lagrange-Hamilton formalism. For symmetric valence and conduction bands $\left|\varepsilon_{\mathrm{v}}(\mathbf{p})\right|=\left|\varepsilon_{\mathrm{c}}(\mathbf{p})\right|$ the freefield Lagrange-Hamilton formalism reduces to the classic KG equation with $\Delta / 2$ effective mass. In general, the operators $\varepsilon_{\mathrm{c}}(\hat{\mathbf{p}})$ and $\varepsilon_{\mathrm{v}}(\hat{\mathbf{p}})$ generate higher order gradients related with possible soliton-type solutions. However, they are beyond the scope of this work.

The next step is to couple the KG oscillator field to the external electromagnetic field. An obstacle in the quantized theory appears because the $\mathrm{KG}$ oscillator field has 4 components while the massless spin-1 photon associated with the external electromagnetic field has only two physical degrees of freedom. Additional conditions that explicitly restrict the number of degrees of freedom to two are introduced within the Coulomb gauge $A^{0}=0$; $\nabla \cdot \mathbf{A}=0$, which, introducing a spin-1 field, leads to the covariant derivative $D_{\mu}=\partial_{\mu}+i e A \mu$ and then, for a charge with its sign included, the substitution $\hat{\mathbf{p}} \rightarrow \hat{\mathbf{P}}=\hat{\mathbf{p}}+e \mathbf{A}[12]$. In accordance with the method of [17], substitution $\hat{\mathbf{p}} \rightarrow \hat{\mathbf{P}}$ will be made in the field function and canonical momenta as given explicitly in the next Section.

\section{Field functions}

Due to higher order gradients in the effective frequency operator, Eq. (9), the construction of field functions deviates from the standard Euler-Lagrange equation technique and requires decomposition of the field function as a sum of plane waves harmonized with the general dispersion law, Eq. (2). Within the decomposition approach the field function is searched as a sum of plane waves:

$$
\Psi(\mathbf{x}, t)=\frac{(2 \pi)^{3 / 2}}{\sqrt{V}} \sum_{\mathbf{p}} \widetilde{\Psi}(\mathbf{p}, t) \mathrm{e}^{\mathrm{ipx}}
$$

where the expansion coefficients $\tilde{\Psi}(\mathbf{p}, \mathrm{t})$ are harmonized with the dispersion relation $\widetilde{\Psi}(E, \mathbf{p})$ by Fourier transformation $\widetilde{\Psi}(\mathbf{p}, t) \equiv \int_{-\infty} \mathrm{d} E \widetilde{\Psi}(E, \mathbf{p}) \mathrm{e}^{-\mathrm{i} E t}$. As a result, the field function, Eq. (17), reads as

$$
\Psi(\mathbf{x}, t)=\frac{(2 \pi)^{3 / 2}}{\sqrt{V}} \sum_{\mathbf{p}}\left(\int_{-\infty}^{\infty} \mathrm{d} E \tilde{\Psi}(E, \mathbf{P}(\mathbf{p}, t)) \mathrm{e}^{-\mathrm{i} E t}\right) \mathrm{e}^{\mathrm{i} \mathbf{p x}}
$$

Here the Fourier transformed expansion coefficients in Eq. (17) are equal to the delta function of dispersion relation $\tilde{\Psi}(E, \mathbf{p})=\delta(g(E, \mathbf{P}))$ and the delta function inside the integral Eq. (18) formally is defined as a distribution function $g(E, \mathbf{P})$.

Finally, the integral in Eq. (18) coincides with the integral form of the generalized scaling property written as

$$
\begin{aligned}
& \int_{-\infty}^{\infty} f(E, \mathbf{p}) \delta(g(E, \mathbf{p})) \mathrm{d} E= \\
& \sum_{i=1,2} \frac{f\left(E_{\mathrm{i}}, \mathbf{p}\right)}{\left|g^{\prime}\left(E_{\mathrm{i}}\right)\right|}=\frac{f\left(E_{1}, \mathbf{p}\right)}{\left|\Delta+\varepsilon_{\mathrm{c}} \mathbf{P}_{\mathrm{c}}(t)+\varepsilon_{\mathrm{v}} \mathbf{P}_{\mathrm{v}}(t)\right|} \\
& +\frac{f\left(E_{2}, \mathbf{p}\right)}{\left|-\Delta-\varepsilon_{\mathrm{c}} \mathbf{P}_{\mathrm{c}}(t)-\varepsilon_{\mathrm{v}} \mathbf{P}_{\mathrm{v}}(t)\right|} .
\end{aligned}
$$

Here $E_{1}=\Delta / 2+\varepsilon_{c}\left(\mathbf{P}_{c}(t)\right)$ and $E_{2}=-\Delta / 2-\varepsilon_{\mathrm{v}}\left(\mathbf{P}_{\mathrm{v}}(t)\right)$ are zeros of the dispersion laws for electrons and holes, correspondingly, and $\mathbf{P}_{\mathrm{c}}=\mathbf{p}+e \mathbf{A}, \mathbf{P}_{\mathrm{v}}=\mathbf{p}-e \mathbf{A}$, and the charge of the electron is set as $e=|e|$.

The functions in numerators are $f\left(E_{\mathrm{i}}, \mathbf{p}\right)=\psi\left(E_{\mathrm{i}} \mathbf{p}\right)$ $\mathrm{e}^{-\mathrm{iEi} t}$, or in an expanded form:

$$
\begin{aligned}
& f\left(E_{1}, \mathbf{p}\right)=\psi\left(\frac{\Delta}{2}+\varepsilon_{\mathrm{c}}\left(\mathbf{P}_{\mathrm{c}}(t)\right)\right) \mathrm{e}^{-\mathrm{i}\left(\frac{\Delta}{2}+\varepsilon_{\mathrm{c}}\left(\mathbf{P}_{\mathrm{c}}(t)\right)\right) t}, \\
& f\left(E_{2}, \mathbf{p}\right)=\psi\left(-\frac{\Delta}{2}-\varepsilon_{\mathrm{v}}\left(\mathbf{P}_{\mathrm{v}}(t)\right)\right) \mathrm{e}^{+\mathrm{i}\left(\frac{\Delta}{2}+\varepsilon_{\mathrm{v}}\left(\mathbf{P}_{\mathrm{v}}(t)\right)\right) t} .
\end{aligned}
$$

The derivative of the dispersion relation reads as $\partial g(E, \mathbf{p}) / \partial E=2 E-\varepsilon_{c}\left(\mathbf{P}_{c}(t)\right)+\varepsilon_{v}\left(\mathbf{P}_{v}(t)\right)$ and determines denominators in the expansion, Eq. (19). Since $\left|g^{\prime}\left(E_{1}\right)\right|=\mid g^{\prime}\left(E_{2}\right)$, it is convenient to introduce an effective frequency

$$
\Omega(\mathbf{P})=\frac{1}{2}\left(\Delta+\varepsilon_{\mathrm{c}}\left(\mathbf{P}_{\mathrm{c}}(t)\right)+\varepsilon_{\mathrm{v}}\left(\mathbf{P}_{\mathrm{v}}(t)\right)\right)
$$

Putting this together one arrives at the field functions harmonized with dispersion laws and represented by sums over the discrete momentum space. The direct field function reads as

$$
\begin{aligned}
& \Psi(\mathbf{x}, t)=\frac{(2 \pi)^{3 / 2}}{\sqrt{V}} \sum_{\mathbf{p}} \frac{1}{\Delta+\varepsilon_{\mathrm{c}}\left(\mathbf{P}_{\mathrm{c}}(t)\right)+\varepsilon_{\mathrm{v}}\left(\mathbf{P}_{\mathrm{v}}(t)\right) \mid} \\
& \times\left(\psi\left(\Delta / 2+\varepsilon_{\mathrm{c}}\left(\mathbf{P}_{\mathrm{c}}(t)\right)\right) \mathrm{e}^{-\mathrm{i}\left(\Delta / 2+\varepsilon_{\mathrm{c}}\left(\mathbf{P}_{\mathrm{c}}(t)\right)\right) t} \mathrm{e}^{\mathrm{i} \mathbf{p x}}\right. \\
& \left.+\psi\left(-\Delta / 2-\varepsilon_{\mathrm{v}}\left(\mathbf{P}_{\mathrm{v}}(t)\right)\right) \mathrm{e}^{+\mathrm{i}\left(\Delta / 2+\varepsilon_{\mathrm{v}}\left(\mathbf{P}_{\mathrm{v}}(t)\right)\right) t} \mathrm{e}^{\mathrm{i} \mathbf{p x}}\right) .
\end{aligned}
$$

The complex conjugate field function of (23) reads as

$$
\begin{aligned}
& \Psi^{*}(\mathbf{x}, t)=\frac{(2 \pi)^{3 / 2}}{\sqrt{V}} \sum_{\mathbf{p}} \frac{1}{\left|\Delta+\varepsilon_{\mathrm{c}}\left(\mathbf{P}_{\mathrm{c}}(t)\right)+\varepsilon_{\mathrm{v}}\left(\mathbf{P}_{\mathrm{v}}(t)\right)\right|} \\
& \times\left(\psi\left(\Delta / 2+\varepsilon_{\mathrm{c}}\left(\mathbf{P}_{\mathrm{c}}(t)\right)\right) \mathrm{e}^{+\mathrm{i}\left(\Delta / 2+\varepsilon_{\mathrm{c}}\left(\mathbf{P}_{\mathrm{c}}(t)\right)\right) t} \mathrm{e}^{-\mathrm{i} \mathbf{p x}}\right. \\
& \left.+\psi\left(-\Delta / 2-\varepsilon_{\mathrm{v}}\left(\mathbf{P}_{\mathrm{v}}(t)\right)\right) \mathrm{e}^{-\mathrm{i}\left(\Delta / 2+\varepsilon_{\mathrm{v}}\left(\mathbf{P}_{\mathrm{v}}(t)\right)\right) t} \mathrm{e}^{-\mathrm{i} \mathbf{p x}}\right) .
\end{aligned}
$$


For subsequent operator representation Eqs. (23), (24) are written as'

$$
\begin{aligned}
& \Psi(\mathbf{x}, t)=\frac{(2 \pi)^{3 / 2}}{\sqrt{V}} \sum_{\mathbf{p}} \frac{1}{\left|\Delta+\varepsilon_{\mathrm{c}}\left(\mathbf{P}_{\mathrm{c}}(t)\right)+\varepsilon_{\mathrm{v}}\left(\mathbf{P}_{\mathrm{v}}(t)\right)\right|} \\
& \times\left(a_{\mathrm{c}}\left(\mathbf{P}_{\mathrm{c}}(t)\right) \mathrm{e}^{-\mathrm{i}\left(\Delta / 2+\varepsilon_{\mathrm{c}}\left(\mathbf{P}_{\mathrm{c}}(t)\right)\right) t}\right. \\
& \left.+a_{\mathrm{v}}\left(\mathbf{P}_{\mathrm{v}}(t)\right) \mathrm{e}^{+\mathrm{i}\left(\Delta / 2+\varepsilon_{\mathrm{v}}\left(\mathbf{P}_{\mathrm{v}}(t)\right)\right) t} \mathrm{e}^{\mathrm{i} \mathbf{p x}}\right), \\
& \Psi^{*}(\mathbf{x}, t)=\frac{(2 \pi)^{3 / 2}}{\sqrt{V}} \sum_{\mathbf{p}} \frac{1}{\left|\Delta+\mathcal{E}_{\mathrm{c}}\left(\mathbf{P}_{\mathrm{c}}(t)\right)+\mathcal{E}_{\mathrm{v}}\left(\mathbf{P}_{\mathrm{v}}(t)\right)\right|} \\
& \times\left(a_{\mathrm{c}}^{*}\left(\mathbf{P}_{\mathrm{c}}(t)\right) \mathrm{e}^{+\mathrm{i}\left(\Delta / 2+\varepsilon_{\mathrm{c}}\left(\mathbf{P}_{\mathrm{c}}(t)\right)\right) t}\right. \\
& \left.+a_{\mathrm{v}}^{*}\left(\mathbf{P}_{\mathrm{v}}(t)\right) \mathrm{e}^{-\mathrm{i}\left(\Delta / 2+\varepsilon_{\mathrm{v}}\left(\mathbf{P}_{\mathrm{v}}(t)\right)\right) t}\right) \mathrm{e}^{\mathrm{i} \mathbf{p x}},
\end{aligned}
$$

With designation accounting for arbitrary dispersion laws $\left|\Delta+\varepsilon_{\mathrm{c}}\left(\mathbf{P}_{\mathrm{c}}(t)\right)+\varepsilon_{\mathrm{v}}\left(\mathbf{P}_{\mathrm{v}}(t)\right)\right|=2 \Omega(\mathbf{P}, t)$ and normalization $a(\mathbf{P}, t) /(V 2 \Omega) \Rightarrow \Delta^{1 / 2} /(V 2 \Omega(\mathbf{P}, t))^{-1 / 2}$ [11], the field functions in operator representation then read:

$$
\begin{aligned}
& \hat{\Psi}(\mathbf{x}, t)=\frac{(2 \pi)^{3 / 2}}{\sqrt{V}} \sum_{\mathbf{p}} \sqrt{\frac{\Delta}{2 \Omega(\mathbf{P}, t)}} \\
& \times\left(\hat{a}_{\mathrm{e}}(\mathbf{P}, t)+\hat{a}_{\mathrm{h}}(\mathbf{P}, t)\right) \mathrm{e}^{\mathrm{i} \mathbf{p x}},
\end{aligned}
$$

or after substitution of appropriate operators as

$$
\begin{aligned}
& \hat{\Psi}^{+}(\mathbf{x}, t)=\frac{(2 \pi)^{3 / 2}}{\sqrt{V}} \sum_{\mathbf{p}} \sqrt{\frac{\Delta}{2 \Omega(\mathbf{P}, t)}} \\
& \times\left(\hat{a}_{\mathrm{e}}^{+}(\mathbf{P}, t)+\hat{a}_{\mathrm{h}}^{+}(\mathbf{P}, t)\right) \mathrm{e}^{-\mathrm{i} \mathbf{p x}} .
\end{aligned}
$$

Equations (27), (28) constitute the background for Lagrange-Hamilton formalism in quasiparticle representation.

\section{Kinetics of electron-hole pair}

The basic object of the kinetic theory of e-h pairs is their distribution function connecting dominant physical features of the single e-h excitation with the many-body description. In a broad sense, the problem is in the description of observable effects of a large number of electrons and nuclei moving in a correlated way in the dielectric solid.

Formally, the kinetics of e-h pairs is distinguished by their distribution functions of electrons and holes [12]

$$
f_{c, v}(\mathbf{p}, t)=\left\langle 0\left|a_{c, v}^{+}(\mathbf{p}, t) a_{c, v}(\mathbf{p}, t)\right| 0\right\rangle,
$$

derived from relations [12]

$$
\begin{aligned}
& \Psi(\mathbf{x}, t)=\frac{(2 \pi)^{3 / 2}}{\sqrt{V}} \sum_{\mathbf{p}} \sqrt{\frac{\Delta}{2 \Omega(\mathbf{P})}}\left\{a_{\mathrm{e}}(\mathbf{p}, t)+a_{\mathrm{h}}(-\mathbf{p}, t)\right\} \mathrm{e}^{\mathrm{i} \mathbf{p x}}, \\
& \pi(\mathbf{x}, t)=\frac{\mathrm{i}(2 \pi)^{3 / 2}}{\sqrt{V}} \sum_{\mathbf{p}} \sqrt{\frac{\Omega(\mathbf{P})}{2 \Delta}}\left\{a_{\mathrm{e}}^{+}(\mathbf{p}, t)-a_{\mathrm{h}}^{+}(-\mathbf{p}, t)\right\} \mathrm{e}^{-\mathrm{i} \mathbf{p x}},
\end{aligned}
$$

where $a_{\mathrm{e}}(\mathbf{p}, \mathrm{t}) \rightarrow a_{\mathrm{e}}(\mathbf{p}) \mathrm{e}^{-\mathrm{i}\left(\varepsilon_{\mathrm{c}}+\Delta / 2\right) t}, a_{\mathrm{h}}(-\mathbf{p}, \mathrm{t}) \rightarrow a_{\mathrm{h}}(-\mathbf{p}) \mathrm{e}^{\mathrm{i}\left(\varepsilon_{\mathrm{c}}+\Delta / 2\right) t}$ and similarly for creation operators [11]. In contrast to Eqs. (23)-(28), the impact of the external field on the field operators is abandoned for simplicity. The total Hamiltonian $H_{\text {tot }}(t)$ comes from free Hamiltonian Eq. (16) [11] and Eqs. (30), (31) as

$$
\begin{aligned}
& H_{\mathrm{tot}}(t)=\frac{(2 \pi)^{3 / 2}}{\sqrt{V}} \sum_{\mathbf{p}}\left\{\left[\varepsilon_{\mathrm{c}}(\mathbf{P})+\frac{\Delta}{2}\right] a_{\mathrm{e}}^{+}(\mathbf{p}) a_{\mathrm{e}}(\mathbf{p})\right. \\
& \left.+\left[\varepsilon_{\mathrm{v}}(-\mathbf{P})+\frac{\Delta}{2}\right] a_{\mathrm{h}}^{+}(-\mathbf{p}) a_{\mathrm{h}}(-\mathbf{p})\right\} .
\end{aligned}
$$

In Eq. (32) the new time-dependent operators $a_{\mathrm{e}, \mathrm{h}}(\mathbf{p}, t)$ obey the exact equations of motion and can be obtained from the principle of least action [11]:

$$
S=\int \mathrm{d} \mathbf{x}\left\{\pi(\mathbf{x}, t) \dot{\Psi}(\mathbf{x}, t) \dot{\Psi}^{*}(\mathbf{x}, t) \pi^{*}(\mathbf{x}, t)-H_{\text {tot }}(\mathbf{x}, t)\right\},
$$

or, explicitly, the action is

$$
\begin{aligned}
& S=\int \mathrm{d} t \frac{(2 \pi)^{3}}{V} \\
& \times\left\{\frac{\mathrm{i}}{2}\left(\begin{array}{l}
{\left[a_{\mathrm{e}}(\mathbf{p}, t)-a_{\mathrm{h}}^{+}(-\mathbf{p}, t)\right]\left[\dot{a}_{\mathrm{e}}(\mathbf{p}, t)+\dot{a}_{\mathrm{h}}(-\mathbf{p}, t)\right]-} \\
{\left[\begin{array}{l}
\left.a_{\mathrm{e}}(\mathbf{p}, t)-a_{\mathrm{h}}(-\mathbf{p}, t)\right]\left[a_{\mathrm{e}}^{+}(\mathbf{p}, t)+\dot{a}_{\mathrm{h}}^{+}(-\mathbf{p}, t)\right]+ \\
\lambda(\mathbf{p}, t) a_{\mathrm{h}}^{+}(-\mathbf{p}, t) a_{\mathrm{e}}(\mathbf{p}, t)-a_{\mathrm{e}}^{+}(\mathbf{p}, t) a_{\mathrm{h}}(-\mathbf{p}, t)
\end{array}\right)-H_{\mathrm{tot}}(\mathbf{p}, t)}
\end{array}\right\} .(34)\right.
\end{aligned}
$$

Here the frequency of interband transitions is given by

$$
\lambda(\mathbf{p}, t)=-\frac{\left(\dot{\varepsilon}_{\mathrm{c}}(\mathbf{P}, t)+\dot{\varepsilon}_{\mathrm{v}}(-\mathbf{P}, t)\right)}{4 \Omega(\mathbf{P}, t)} .
$$

Kinetics for the electron and hole operators are obtained from Eq. (34) by minimum action principle that yields two pairs of differential equations. The first pair, Eq. (36), is for the electron/hole annihilation operators, the second pair is complex conjugate and describes kinetics for the electron/hole annihilation:

$$
\left\{\begin{array}{l}
\dot{a}_{\mathrm{e}}(\mathbf{p}, t)=\lambda(\mathbf{p}, t) a_{\mathrm{h}}^{+}(-\mathbf{p}, t)+\mathrm{i}\left[H_{\mathrm{tot}}(t), a_{\mathrm{e}}(\mathbf{p}, t)\right] \\
\dot{a}_{\mathrm{h}}(\mathbf{p}, t)=\lambda(\mathbf{p}, t) a_{\mathrm{e}}^{+}(-\mathbf{p}, t)+\mathrm{i}\left[H_{\mathrm{tot}}(t), a_{\mathrm{h}}(\mathbf{p}, t)\right] .
\end{array}\right.
$$

The first term in the right hand side of Eq. (36) describes intermixing of the electron and hole states. It is assumed here that the system is found in the 
vacuum state $|0\rangle$ of electrons and holes at the initial momenta $t_{0} \rightarrow-\infty$.

The general vacuum state found by differentiating the distribution function, Eq. (29), with respect to time and using equations of motion, Eq. (36), [12] reads as

$$
\dot{f}_{c, v}(\mathbf{p}, t)=\lambda(\mathbf{p}, t)\left\{f_{c, v}(\mathbf{p}, t)+f_{\mathrm{v}, \mathrm{c}}(\mathbf{p}, t)\right\},
$$

where the auxiliary correlation functions are introduced:

$$
\begin{aligned}
& f_{\mathrm{cv}}(\mathbf{p}, t)=\left\langle 0\left|a_{\mathrm{c}}^{+}(\mathbf{p}, t) a_{\mathrm{v}}(\mathbf{p}, t)\right| 0\right\rangle, \\
& f_{\mathrm{vc}}(\mathbf{p}, t)=\left\langle 0\left|a_{\mathrm{v}}^{+}(\mathbf{p}, t) a_{\mathrm{c}}(\mathbf{p}, t)\right| 0\right\rangle .
\end{aligned}
$$

The equations of motion for auxiliary correlation functions (38) are obtained in the integral form:

$$
\begin{aligned}
& f_{\mathrm{cv}}^{( \pm)}(\mathbf{p}, t)=\int_{-\infty}^{t} \mathrm{~d} t^{\prime} \lambda\left(\mathbf{p}, t^{\prime}\right) \\
& \times\left[1-f_{\mathrm{e}}\left(\mathbf{p}, t^{\prime}\right)-f_{\mathrm{h}}\left(-\mathbf{p}, t^{\prime}\right)\right] \mathrm{e}^{ \pm 2 i \theta(}\left(\mathbf{p}, t^{\prime}\right),
\end{aligned}
$$

where now $f_{\mathrm{c}}=f_{\mathrm{e}}$ and $f_{\mathrm{v}}=1-f_{\mathrm{h}}, f_{\mathrm{e}}$ and $f_{\mathrm{h}}$ are the electron and hole distribution functions. In Eq. (39) the following initial condition has been introduced:

$$
\lim _{t \rightarrow-\infty} f_{\mathrm{cv}}^{( \pm)}(\mathbf{p}, t)=0 .
$$

Then the kinetic equation for the e-h quasiparticle distribution function [12] reads as

$$
\begin{aligned}
& \dot{f}(\mathbf{p}, t)=2 \lambda(\mathbf{p}, t) \int_{-\infty}^{t} \mathrm{~d} t^{\prime} \lambda\left(\mathbf{p}, t^{\prime}\right) \\
& \times\left[1-2 f\left(\mathbf{p}, t^{\prime}\right)\right] \cos 2 \theta\left(\mathbf{p}, t, t^{\prime}\right),
\end{aligned}
$$

where the dynamical phase is given by $\theta\left(\mathbf{p}, t, t^{\prime}\right)=$ $\int_{t^{\prime}}^{t} \mathrm{~d} \tau \Omega(\mathbf{P}, \tau)[11]$.

In Eq. (41) we follow the notation of [11], and the change of variables $\mathbf{p} \rightarrow \mathbf{P}$ was not implemented explicitly.

The densities of observables follow from the quasiparticle distribution functions of electrons and holes by rewriting Eq. (41) in a gauge invariant form, changing variables $\mathbf{p} \rightarrow \mathbf{P}$ in the distribution functions $f(\mathbf{p}, t) \rightarrow f(\mathbf{P}, t)$, and averaging over the vacuum state of the corresponding operators [11].

The derived quantities of the kinetic theory of e-h pairs include densities of total energy, carrier (e-h pair) number, and total current [12] $\left([\mathrm{d} p]=(2 \pi)^{-3} \mathrm{~d}^{3} p\right)$ :

$$
\begin{aligned}
& \omega(t)=2 \int[\mathrm{d} \mathbf{p}][2 \varepsilon(\mathbf{p}, t)+\Delta] f(\mathbf{p}, t) \\
& n(t)=4 \int[\mathrm{d} \mathbf{p}] f(\mathbf{p}, t) \\
& \mathbf{j}(t)=\frac{4 e}{m} \int[\mathrm{d} \mathbf{p}] \mathbf{p}\{f(\mathbf{p}, t)+u(\mathbf{p}, t)\}=\mathbf{j}_{\text {cond }}(t)+\mathbf{j}_{\mathrm{pol}}(t),
\end{aligned}
$$

where $u(\mathbf{p}, t)$ may be found from solution of Eq. (41) as $\dot{f}=\lambda u$ as a result of numerical analysis. Equations (42)-(44) are written in a thermodynamic limit $V \rightarrow \infty$ [12].

It is important to observe that this form of KG formalism has been developed earlier in the study of ultrafast and strong field interband transitions [11, 12].

Complementary approaches to this $\mathrm{KG}$ formalism include approximate solutions for Hamiltonian systems structured of a non-interacting and a driving term. Examples are an atom in the electromagnetic field (dipole approximation) and its simplification for light frequency presumably close to the energy difference between two states of the atom.

In another, rotating wave approximation (RWA), the radiation field is formally split in a product of a slow-varying envelope and a fast-oscillating harmonic function. RWA gives solutions of Schrödingertype problems in an appropriate analytic form. However, the conditions for the RWA to be valid are (i) limited by the spectral width of the light pulse much less than the light frequency, (ii) the light frequency is near-resonant with the transition frequency, and (iii) the Rabi frequency is much less than the transition frequency.

A successful example of RWA is elaborated in [9] for the dynamics of electrons and holes in a semiconductor with their interaction described by the e-h pair dynamics similarly to that in Eq. (4). In spite of standard limitations of the perturbation technique, the SC approach [9] captures a rich scope of properties of the e-h pairs and excitons, including their deviation from the composite boson state. Otherwise, the KG formalism is nonperturbative in its nature, allows to calculate the non-equilibrium distribution and all relevant derived quantities. Yet, to use its potential completely, the e-h interaction with other fields is highly motivated.

Our contribution is to employ the KG formalism in conjunction with the back reaction of the phonon subsystem to the formation of the electron-hole subsystem. It is expected that this will allow more realistic exploration of phonon-assisted kinetics of e-h pair creation in solids.

\section{Electron-phonon and hole-phonon interaction}

Many-particle models unavoidably lead to the problem of particle interaction. Examples include electron-nucleus interaction described by a combination of Dirac and KG equations [18] and plasma oscillations making use of the quantum Vlasov equation [19]. In contrast, the mathematical technique addressed to the photoexcited kinetics of electrons and holes in lattice systems 
is different and the effect of interaction is treated here making use of a supplementary interaction Hamiltonian that modifies the total Hamiltonian Eq. (32) by the back reaction term. In this Section, the reduced Planck constant $\hbar$ is restored.

For the system consisting of e-h and phonons we redefine the total electron-hole Hamiltonian Eq. (32) by adding the phonon and particle-phonon interaction terms:

$$
\begin{aligned}
& \mathrm{H}=\sum_{\mathbf{p}} E_{\mathrm{c}}(\mathbf{p}) a_{\mathrm{e}}^{+}(\mathbf{p}) a_{\mathrm{e}}(\mathbf{p}) \\
& +\sum_{\mathbf{p}} E_{\mathrm{v}}(\mathbf{p}) a_{\mathrm{h}}^{+}(\mathbf{p}) a_{\mathrm{h}}(\mathbf{p})+\sum_{\mathbf{q}} \hbar \omega_{\mathbf{q}} c_{\mathbf{q}}^{+} c_{\mathbf{q}} \\
& +\sum_{\mathbf{p q}} V_{(\mathrm{e}) \mathbf{q}}\left(a_{\mathrm{e}, \mathbf{p}+\mathbf{q}}^{+} a_{\mathrm{e}, \mathbf{q}} c_{\mathbf{q}}+c_{\mathbf{q}}^{+} a_{\mathrm{e}, \mathbf{p}-\mathbf{q}}^{+} a_{\mathbf{q}}\right) \\
& +\sum_{\mathbf{p q}} V_{(\mathrm{h}) \mathbf{q}}\left(a_{\mathrm{h}, \mathbf{p}+\mathbf{q}}^{+} a_{\mathrm{h}, \mathbf{q}} c_{\mathbf{q}}+c_{\mathbf{q}}^{+} a_{\mathrm{h}, \mathbf{p}-\mathbf{q}}^{+} a_{\mathbf{q}}\right),
\end{aligned}
$$

where $\hbar \omega_{q}$ is phonon energy with momentum $\mathbf{q}$ and $\left\{c_{\mathrm{q}}^{+}, c_{\mathrm{q}}\right\}$ are respective phonon creation and annihilation operators. The dispersion of electrons and holes are $E_{\mathrm{c}}(\mathbf{p})=\Delta / 2+\varepsilon_{\mathrm{c}}(\mathbf{p})$ and $E_{\mathrm{v}}(\mathbf{p})=-\Delta / 2-\varepsilon_{\mathrm{c}}(\mathbf{p})$, and $V_{(\mathrm{e}, \mathrm{h}) \mathbf{q}}$ are matrix elements weighting the combinations of creation and annihilation operators [13].

For further application of $\mathrm{BCH}$ it is convenient to rewrite Eq. (45) in the sum of a bare Hamiltonian and particle-phonon interaction Hamiltonians:

$$
H=H_{0}+H_{1}^{\mathrm{e}}+H_{1}^{\mathrm{h}} \text {. }
$$

Here, the bare Hamiltonian reads as

$$
H_{0}=\sum_{\mathbf{p}} E_{\mathrm{c}}(\mathbf{p}) a_{\mathrm{e}}^{+}(\mathbf{p}) a_{\mathrm{e}}(\mathbf{p})+\sum_{\mathbf{p}} E_{\mathrm{v}}(\mathbf{p}) a_{\mathrm{h}}^{+}(\mathbf{p}) a_{\mathrm{h}}(\mathbf{p}) .
$$

To focus on the e-h subsystem we decouple the electron-phonon and hole-phonon interaction by means of $\mathrm{BCH}$ canonical transformation

$$
\widetilde{H}=\mathrm{e}^{-S} H \mathrm{e}^{S}=H+[H, S]+\frac{1}{2}[[H, S], S]+\ldots,
$$

where $S$ is a generator of the transformation (must not be confused with the action in Eq. (34)). Substitution of Eq. (46) into Eq. (48) redefines the $H_{1}$ term of Hamiltonian Eq. (46) as

$$
\begin{aligned}
& \tilde{H}=H+[H, S]+\ldots=H_{0}+H_{1}^{\mathrm{e}}+H_{1}^{\mathrm{h}} \\
& +\left[H_{0}+H_{1}^{\mathrm{e}}+H_{1}^{\mathrm{h}}, S\right]=H_{0}+\left[H_{1}^{\mathrm{e}}+H_{1}^{\mathrm{h}}, S\right] .
\end{aligned}
$$

The next step [16] is to eliminate linear electronhole-phonon interaction by condition

$$
H_{1}^{\mathrm{e}}+H_{1}^{\mathrm{h}}+\left[H_{0}, S\right]=0 \text {. }
$$

Then, cancelling higher orders of $S$ in Eq. (48), we obtain in the second order perturbation approach

$$
\frac{1}{2}[[H, S], S]=-\frac{1}{2}\left[H_{1}^{\mathrm{e}}+H_{1}^{\mathrm{h}}, S\right] .
$$

Substitution of Eq. (51) back into Eq. (49) gives the expected $\mathrm{BCH}$ as

$$
\widetilde{H}=H_{0}+\frac{1}{2}\left[H_{1}^{\mathrm{e}}+H_{1}^{\mathrm{h}}, S\right]=H_{0}+H_{\text {int }} .
$$

The $\mathrm{BCH}$ transformed Hamiltonian Eq. (52) contains two new entities - the generator of transformation $S$ and the interaction Hamiltonian $H_{\text {int }}$. Calculation of the matrix elements of $S$ in the basis of eigenfunctions of a nonperturbed Hamiltonian $H_{0}|n\rangle=E_{n}|n\rangle$ (here $E_{n}$ are eigenvalues of a nonperturbed (bare) Hamiltonian) and making use of Eq. (50) gives the relation

$$
\begin{aligned}
& \left\langle m\left|H_{1}^{\mathrm{e}}\right| n\right\rangle+\left\langle m\left|H_{1}^{\mathrm{h}}\right| n\right\rangle \\
& +\left\langle m\left|H_{0} S\right| n\right\rangle-\left\langle m\left|S H_{0}\right| n\right\rangle=0 .
\end{aligned}
$$

The third and fourth terms of Eq. (53) generate products of matrix elements

$$
\begin{aligned}
& \left\langle m\left|H_{0} S\right| n\right\rangle=\sum_{i}\left\langle m\left|H_{0}\right| i\right\rangle\langle i|S| n\rangle= \\
& \sum_{i} E_{m}\langle m|S| n\rangle, \\
& \left\langle m\left|S H_{0}\right| n\right\rangle=\sum_{i}\langle m|S| i\rangle\left\langle i\left|H_{0}\right| n\right\rangle= \\
& \sum_{i} E_{n}\langle m|S| n\rangle .
\end{aligned}
$$

Putting together Eqs. (54) and (55) gives the matrix element of $S$ as

$$
\langle m|S| n\rangle=\frac{\left\langle m\left|H_{1}^{\mathrm{e}}\right| n\right\rangle+\left\langle m\left|H_{1}^{\mathrm{h}}\right| n\right\rangle}{E_{n}-E_{m}} .
$$

Similarly, the matrix elements of $H_{\text {int }}$ follow from the relation in Eq. (52) as

$$
\begin{aligned}
& \left\langle m\left|H_{\text {int }}\right| n\right\rangle=\frac{1}{2}\left(\left\langle m\left|H_{1}^{\mathrm{e}} S\right| n\right\rangle+\left\langle m\left|H_{1}^{\mathrm{h}} S\right| n\right\rangle\right. \\
& \left.-\left\langle m\left|H_{1}^{\mathrm{e}} S\right| n\right\rangle-\left\langle m\left|S H_{1}^{\mathrm{h}}\right| n\right\rangle\right)=\frac{1}{2}(a+b+c+d),
\end{aligned}
$$

where the following notation was introduced:

$$
\begin{aligned}
& a=\sum_{g}\left\langle m\left|H_{1}^{\mathrm{e}}\right| g\right\rangle\langle g|S| n\rangle= \\
& \sum_{g}\left\langle m\left|H_{1}^{\mathrm{e}}\right| g\right\rangle \frac{\left\langle g\left|H_{1}^{\mathrm{e}}\right| n\right\rangle+\left\langle g\left|H_{1}^{\mathrm{h}}\right| n\right\rangle}{E_{n}-E_{g}}, \\
& b=\sum_{g}\left\langle m\left|H_{1}^{\mathrm{h}}\right| g\right\rangle\langle g|S| n\rangle= \\
& \sum_{g}\left\langle m\left|H_{1}^{\mathrm{h}}\right| g\right\rangle \frac{\left\langle g\left|H_{1}^{\mathrm{e}}\right| n\right\rangle+\left\langle g\left|H_{1}^{\mathrm{h}}\right| n\right\rangle}{E_{n}-E_{g}},
\end{aligned}
$$




$$
\begin{aligned}
& c=-\sum_{g}\langle m|S| g\rangle\left\langle g\left|H_{1}^{\mathrm{e}}\right| n\right\rangle= \\
& \sum_{g} \frac{\left\langle m\left|H_{1}^{\mathrm{e}}\right| g\right\rangle+\left\langle m\left|H_{1}^{\mathrm{h}}\right| g\right\rangle}{E_{g}-E_{m}}\left\langle g\left|H_{1}^{\mathrm{e}}\right| n\right\rangle, \\
& d=-\sum_{g}\langle m|S| g\rangle\left\langle g\left|H_{1}^{\mathrm{h}}\right| n\right\rangle= \\
& \sum_{g} \frac{\left\langle m\left|H_{1}^{\mathrm{e}}\right| g\right\rangle+\left\langle m\left|H_{1}^{\mathrm{h}}\right| g\right\rangle}{E_{g}-E_{m}}\left\langle g\left|H_{1}^{\mathrm{h}}\right| n\right\rangle .
\end{aligned}
$$

Going back to the matrix of the interaction Hamiltonian Eq. (57) we find the following constituents of the electron-phonon and hole-phonon interaction due to the exchange of virtual phonons:

$$
\begin{aligned}
& e=\frac{1}{2} \sum_{g}\left\langle m\left|H_{1}^{\mathrm{e}}\right| g\right\rangle\left\langle g\left|H_{1}^{\mathrm{e}}\right| n\right\rangle \\
& \times\left(\frac{1}{E_{n}-E_{g}}+\frac{1}{E_{g}-E_{m}}\right), \\
& h=\sum_{g}\left\langle m\left|H_{1}^{\mathrm{h}}\right| g\right\rangle\left\langle g\left|H_{1}^{\mathrm{h}}\right| n\right\rangle \\
& \times\left(\frac{1}{E_{n}-E_{g}}+\frac{1}{E_{g}-E_{m}}\right), \\
& h e=\sum_{g}\left\langle m\left|H_{1}^{\mathrm{h}}\right| g\right\rangle\left\langle g\left|H_{1}^{\mathrm{e}}\right| n\right\rangle \\
& \times\left(\frac{1}{E_{g}-E_{m}}+\frac{1}{E_{n}-E_{g}}\right), \\
& e h=\sum_{g}\left\langle m\left|H_{1}^{\mathrm{e}}\right| g\right\rangle\left\langle g\left|H_{1}^{\mathrm{h}}\right| n\right\rangle \\
& \times\left(\frac{1}{E_{n}-E_{g}}+\frac{1}{E_{g}-E_{m}}\right) .
\end{aligned}
$$

Here, the sums run over momenta $\mathbf{p}, \mathbf{q}$ and interaction factors $V_{(e, h)}$, Eq. (45), are temporarily omitted. If there are no holes, then $H_{1}^{\mathrm{h}}=0$, and only the electron-phonon interaction remains. The particlephonon interaction terms, Eq. (45), constituting the matrix elements in Eqs. (59)-(62) read as

$$
\begin{aligned}
& H_{1}^{\mathrm{e}}=\sum_{\mathbf{p q}} V_{(\mathrm{e}) \mathbf{q}}\left(a_{\mathrm{e}, \mathbf{p}+\mathbf{q}}^{+} a_{\mathrm{e}, \mathbf{p}} c_{\mathbf{q}}+c_{\mathbf{q}}^{+} a_{\mathrm{e}, \mathbf{p}-\mathbf{q}}^{+} a_{\mathrm{e}, \mathbf{p}}\right), \\
& H_{1}^{\mathrm{h}}=\sum_{\mathbf{p q}} V_{(\mathrm{h}) \mathbf{q}}\left(a_{\mathrm{h}, \mathbf{p}+\mathbf{q}}^{+} a_{\mathrm{h}, \mathbf{p}} c_{\mathbf{q}}+c_{\mathbf{q}}^{+} a_{\mathrm{h}, \mathbf{p}-\mathbf{q}}^{+} a_{\mathrm{h}, \mathbf{p}}\right),
\end{aligned}
$$

where $a_{\mathrm{e}, \mathrm{h}}^{+}$and $a_{\mathrm{e}, \mathrm{h}}$ are operators that create/annihilate electrons and holes, correspondingly. These operators are known entities that can be obtained from Eq. (36). The operators $c^{+}$and $c$ are assigned to phonons. Detailed calculations are addressed to interactions in Eqs. (59)(62) where the states $\langle m|$ and $|n\rangle$ contain no phonons.
We shall illustrate the regular procedure by calculating the electron-phonon interaction in Eq. (59). Substitution of Eq. (63) in Eq. (59) and neglecting the interaction factor $V_{(\mathrm{e}) \mathbf{q}}$ and summing over $\mathbf{p}, \mathbf{q}$ and $g$ give two matrix elements:

$$
\begin{aligned}
& e_{1}=\left\langle m\left|H_{1}^{\mathrm{e}}\right| g\right\rangle\left\langle g\left|H_{1}^{\mathrm{e}}\right| n\right\rangle \frac{1}{2} \\
& \times\left(\frac{1}{E_{n}-E_{g}}+\frac{1}{E_{g}-E_{m}}\right) .
\end{aligned}
$$

The first matrix element,

$$
\begin{aligned}
& \boldsymbol{e}_{11}=\left\langle m\left|a_{\mathrm{e}, \mathbf{p}+\mathbf{q}}^{+} a_{\mathrm{e}, \mathbf{p}^{\prime}} c_{\mathbf{q}}+c_{\mathbf{q}_{\mathrm{e}, \mathbf{p}^{\prime} \mathbf{q}}^{+} a_{\mathrm{e}, \mathbf{p}}^{+}}\right| g\right\rangle= \\
& \left\langle m\left|a_{\mathrm{e}, \mathbf{p}^{\prime}+\mathbf{q}}^{+} a_{\mathrm{e}, \mathbf{p}^{\prime}}, c_{\mathbf{q}}\right| g\right\rangle,
\end{aligned}
$$

creates an electron with momentum $\mathbf{p}^{\prime}+\mathbf{q}$, destroys (annihilates) an electron with momentum $\mathbf{p}^{\prime}$, and destroys a phonon with momentum q. The difference between states is

$$
E_{\mathrm{el}}\left(\mathbf{p}^{\prime}+\mathbf{q}\right)-E_{\mathrm{el}}(\mathbf{p})-\hbar \omega_{\mathbf{q}} .
$$

The second matrix element,

$$
\begin{aligned}
& e_{12}=\left\langle g\left|a_{\mathrm{e}, \mathbf{p}+\mathbf{q}}^{+} a_{\mathrm{e}, \mathbf{p}} c_{\mathbf{q}}+c_{\mathbf{q}}^{+} a_{\mathrm{e}, \mathbf{p}-\mathbf{q}}^{+} a_{\mathrm{e}, \mathbf{p}}\right| n\right\rangle= \\
& \left\langle g\left|c_{\mathbf{q}}^{+} a_{\mathrm{e}, \mathbf{p}-\mathbf{q}}^{+} a_{\mathrm{e}, \mathbf{p}}\right| n\right\rangle,
\end{aligned}
$$

creates an electron with momentum $\mathbf{p}-\mathbf{q}$, destroys an electron with momentum $\mathbf{p}$, and creates a phonon with momentum q. The difference between states is

$$
-E_{\mathrm{el}}(\mathbf{p}-\mathbf{q})+E_{\mathrm{el}}(\mathbf{p})-\hbar \omega_{\mathbf{q}} .
$$

Using Eqs. (66)-(68) and restoring the interaction factor $V_{(e) q}$ and sums over $\mathbf{p}, \mathbf{q}$ the electron-phonon interaction in Eq. (59) reads as

$$
\begin{aligned}
& e=\sum_{\mathbf{p}, \mathbf{p}^{\prime}, \mathbf{q}} \frac{V_{\mathrm{e}, \mathbf{q}}^{2}}{2}\left(\frac{1}{E\left(\mathbf{p}^{\prime}+\mathbf{q}\right)-E\left(\mathbf{p}^{\prime}\right)-\hbar \omega_{\mathbf{q}}}\right. \\
& \left.+\frac{1}{E(\mathbf{p})-E(\mathbf{p}-\mathbf{q})-\hbar \omega_{\mathbf{q}}}\right) a_{\mathrm{e}, \mathbf{p}^{+}+\mathbf{q}}^{+} a_{\mathrm{e}, \mathbf{p}^{\prime}} a_{\mathrm{e}, \mathbf{p}-\mathbf{q}}^{+} a_{\mathrm{e}, \mathbf{p}} .
\end{aligned}
$$

Calculations similar to those in Eqs. (65) $-(70)$ yield

$$
\begin{aligned}
& e h=\sum_{\mathbf{p}, \mathbf{p}^{\prime}, \mathbf{q}} \frac{V_{\mathrm{e}, \mathbf{q}} V_{\mathrm{h}, \mathbf{q}}}{2}\left(\frac{1}{E\left(\mathbf{p}^{\prime}+\mathbf{q}\right)-E\left(\mathbf{p}^{\prime}\right)-\hbar \omega_{\mathbf{q}}}\right. \\
& \left.+\frac{1}{-E(\mathbf{p}-\mathbf{q})+E(\mathbf{p})-\hbar \omega_{\mathbf{q}}}\right) a_{\mathrm{e}, \mathbf{p}^{\prime} \mathbf{q}}^{+} a_{\mathrm{e}, \mathbf{p}^{\mathrm{p}}} a_{\mathrm{h}, \mathbf{p}-\mathbf{q}}^{+} a_{\mathrm{h}, \mathbf{p}} . \\
& h=\frac{\left(V_{\mathrm{h}, \mathbf{q}}\right)^{2}}{2}\left(\frac{1}{E\left(\mathbf{p}^{\prime}+\mathbf{q}\right)-E\left(\mathbf{p}^{\prime}\right)-\hbar \omega_{\mathbf{q}}}\right. \\
& \left.+\frac{1}{-E(\mathbf{p}-\mathbf{q})+E(\mathbf{p})-\hbar \omega_{\mathbf{q}}}\right) a_{\mathrm{h}, \mathbf{p}^{\prime}+\mathbf{q}}^{+} a_{\mathrm{h}, \mathbf{p}^{\prime}} a_{\mathrm{h}, \mathbf{p}-\mathbf{q}}^{+} a_{\mathrm{h}, \mathbf{p}},
\end{aligned}
$$




$$
\begin{aligned}
& h e=\sum_{\mathbf{p}, \mathbf{p}^{\prime}, \mathbf{q}} \frac{V_{\mathrm{e}, \mathbf{q}} V_{\mathrm{h}, \mathbf{q}}}{2}\left(\frac{1}{E\left(\mathbf{p}^{\prime}+\mathbf{q}\right)-E\left(\mathbf{p}^{\prime}\right)-\hbar \omega_{\mathrm{q}}}\right. \\
& \left.+\frac{1}{-E(\mathbf{p}-\mathbf{q})+E(\mathbf{p})-\hbar \omega_{\mathbf{q}}}\right) a_{\mathrm{e}, \mathbf{p}^{\prime}+\mathbf{q}}^{+} a_{\mathrm{e}, \mathbf{p}^{\prime}} a_{\mathrm{h}, \mathbf{p}-\mathbf{q}}^{+} a_{\mathrm{h}, \mathbf{p}} .
\end{aligned}
$$

The energy conservation law $E\left(\mathbf{p}^{\prime}+\mathbf{q}\right)-E\left(\mathbf{p}^{\prime}\right)=$ $-E(\mathbf{p})+E(\mathbf{p}-\mathbf{q})$ substituted in Eqs. (70) $-(73)$ represents the effective interaction in form [14]

$$
\begin{aligned}
& e=\sum_{\mathbf{p}, \mathbf{p}^{\prime}, \mathbf{q}} V_{\mathrm{e}, \mathbf{q}}^{2}\left(\frac{\hbar \omega_{\mathbf{q}}}{(E(\mathbf{p}-\mathbf{q})-E(\mathbf{p}))^{2}-\left(\hbar \omega_{\mathbf{q}}\right)^{2}}\right) \\
& \times a_{\mathrm{e}, \mathbf{p}^{\prime}+\mathbf{q}}^{+} a_{\mathrm{e}, \mathbf{p}^{\prime}} a_{\mathrm{e}, \mathbf{p}-\mathbf{q}}^{+} a_{\mathrm{e}, \mathbf{p}} \\
& e h=\sum_{\mathbf{p}, \mathbf{p}^{\prime}, \mathbf{q}} V_{\mathrm{e}, \mathbf{q}} V_{\mathrm{h}, \mathbf{q}}\left(\frac{\hbar \omega_{\mathbf{q}}}{(E(\mathbf{p}-\mathbf{q})+E(\mathbf{p}))^{2}-\left(\hbar \omega_{\mathbf{q}}\right)^{2}}\right) \\
& \times a_{\mathrm{e}, \mathbf{p}^{\prime}+\mathbf{q}}^{+} a_{\mathrm{e}, \mathbf{p}^{\prime}} a_{\mathrm{h}, \mathbf{p}-\mathbf{q}}^{+} a_{\mathrm{h}, \mathbf{p}}, \\
& h=\sum_{\mathbf{p}, \mathbf{p}^{\prime}, \mathbf{q}}\left(V_{\mathrm{h}, \mathbf{q}}\right)^{2}\left(\frac{\hbar \omega_{\mathbf{q}}}{(E(\mathbf{p}-\mathbf{q})+E(\mathbf{p}))^{2}-\left(\hbar \omega_{\mathbf{q}}\right)^{2}}\right) \\
& \times a_{\mathrm{h}, \mathbf{p}^{\prime}+\mathbf{q}}^{+} a_{\mathrm{h}, \mathbf{p}^{\prime}} a_{\mathrm{h}, \mathbf{p}-\mathbf{q}}^{+} a_{\mathrm{h}, \mathbf{p}}, \\
& h e=\sum_{\mathbf{p}, \mathbf{p}^{\prime}, \mathbf{q}} V_{\mathrm{h}, \mathbf{q}} V_{\mathrm{e}, \mathbf{q}}\left(\frac{\hbar \omega_{\mathbf{q}}}{(E(\mathbf{p}-\mathbf{q})+E(\mathbf{p}))^{2}-\left(\hbar \omega_{\mathbf{q}}\right)^{2}}\right) \\
& \times a_{\mathrm{h}, \mathbf{p}+\mathbf{q}}^{+} a_{\mathrm{h}, \mathbf{p}} a_{\mathrm{e}, \mathbf{p}-\mathbf{q}}^{+} a_{\mathrm{e}, \mathbf{p}} .
\end{aligned}
$$

Finally, the interaction Hamiltonian that specifies the back reaction of phonons is given by the sum of particular interactions in Eqs. (74)-(77) as

$$
H_{\text {int }}=e+h+h e+e h,
$$

where the creation and annihilation operators of electrons and holes $a_{\mathrm{e}}^{+}, a_{\mathrm{e}}, a_{\mathrm{h}}^{+}, a_{\mathrm{h}}$ as well as the dispersions $E(\mathbf{p})$ are known momentum- and time-dependent entities. The interaction Hamiltonian and the frequencies of interband transitions can be obtained from Eqs. (34) and (35) as

$$
H_{\text {tot }} \rightarrow H_{\text {tot }}+H_{\text {int }}
$$

As a result, the canonical approach to strong field interband transitions [11] is maintained both in its QFT and QED parts. An exception appears at the minimal action stage, Eq. (34), in which the total Hamiltonian Eq. (32) is supplemented by the interaction Hamiltonian Eq. (78). The operator equations of motion follow from Eqs. (34) and (79) by variation on the amplitudes and subsequent transition to the occupation number representation with anticommutation relations $\left[a_{0,1}(\mathbf{p}), a_{\mathrm{e}, \mathrm{k}}^{+}(\mathbf{q})\right]=\delta$ and equations of motion, Eqs. (63) and (64) [11], calculations of distribution functions, Eqs. (41) and (42) as well as the derived quantities (densities of total energy, Eq. (42)), carrier (e-h pair) number, Eq. (43), and total current, Eq. (44).

General definitions for optical response start with the connection between polarization current and dipole momentum density as [12]

$$
\mathbf{j}_{\text {pol }}(t)=\dot{\mathbf{P}}(t) \text {. }
$$

For stationary processes Eq. (80) leads to the optical susceptibility and the absorption coefficient defined by its imaginary part. However, the quantum kinetic theory, which combines the dispersion properties of both electron and hole, Eq. (1), is addressed especially to the e-h states and relevant physical quantities far from equilibrium.

The resulting kinetics contains two time scales: one associated with the external field and another with the phonon frequencies. Their coupling leads to a very complicated structure of the distribution function and is of interest for a more adequate description of a non-parabolic dispersion, observables under strong fields far from equilibrium, and for joint QED and SC approaches.

\section{Conclusions}

In this paper, we obtained a simple Baker-CampbellHausdorf $(\mathrm{BCH})$-type solution to the phonon-assisted kinetics of the electron-hole pair in a two-band model of dielectric solids. The mathematical background is focussed on the Klein-Gordon-type nonperturbative kinetics in a strong electric field and electron-hole excitation. The results may be useful in describing the effect of femptosecond electromagnetic radiation, impact of phonons, and consequences for observable effects.

The extension of a prototypic approach to strong field interband transitions is addressed to the back reaction of phonons within the $\mathrm{BCH}$ canonical transformation that disconnects the electron-phonon and hole-phonon interaction to a purely electron-hole problem available for implementation in the quantum electrodynamics formalism. The output is a unifying description for the distribution function of electron and hole quasiparticles on the first, excitation, stage of their evolution. Extension of the present solution to the second, relaxation, stage after termination of radiation requires implementation of electrostatic interaction and will be presented in a separate work.

The formalism described above is of interest for fine features of optical spectra under high-intensity 
short-pulse laser radiation and for further developments to find a rationale from the joint quantum electrodynamics formalism and semiconductor approaches, namely, lift the limitations to the electromagnetic field in the semiconductor approach and introduce electrostatic interaction in the quantum electrodynamics formalism.

\section{Acknowledgements}

The author expresses his deep gratitude to A.I. Popov and G. Zvejnieks for inspiring comments and for critical reading of the manuscript. This work was supported in part by the National Research Program of Latvia No. 2014. 10-4/VPP-2/ and Grant No. $237 / 2012$.

\section{References}

[1] M. Agrawal, Quantum field theory (QFT) and quantum optics (QED), in: Fundamental Physics in Nano-Structured Materials and Devices (Stanford University, 2008).

[2] H. Fröhlich, Electrons in lattice fields, Adv. Phys. 3, 325 (1954).

[3] J. Fröhlich, Existence of dressed one electron states in a class of persistent models, Fortschr. Phys. 22, 159 (1974).

[4] T. Holstein, Studies of polaron motion: Part I. The molecular-crystal model, Ann. Phys. (N.Y.) 8, 325(1959).

[5] T. Holstein, Studies of polaron motion: Part II. The "small" polaron, Ann. Phys. (N.Y.) 8, 343 (1959).

[6] A.M. Yaremko, V.M. Dzhagan, V.O. Yukhuymchuk, T.L. Linnik, H. Ratajczak, J. Baran, and A.J. Barnes, Many particle approach to excitons in crystals: electron-electron and electron-phonon interactions, J. Mol. Struct. 976, 205-214 (2010), http:// dx.doi.org/10.1016/j.molstruc.2010.03.086

[7] K. Hyeon-Deuk and O.V. Prezhdo, I. Phys.: Condens. Matter 24, 363201 (2012), http://dx.doi. org/10.1088/0953-8984/24/36/363201

[8] P. Han and G. Bester, First-principles calculation of the electron-phonon interaction in semiconductor nanoclusters, Phys. Rev. B 85, 235422 (2012), http://dx.doi.org/ 10.1103/PhysRevB.85.235422
[9] M. Erementchouk, V. Turkowski, and M.N. Leuenberger, Quantum field theory of exciton correlations and entanglement in semiconductor structures, in: Advances in Quantum Field Theory, ed. S. Ketov, Chapter 6, ISBN 978-953-51-0035-5, http://dx.doi.org/10.5772/37699, published 3 February 2012 under CC BY 3.0 license.

[10] A.V. Friesen, A.V. Prozorkevich, S.A. Smolyansky, and M. Bonitz, Nonperturbative kinetics of electron-hole excitations in strong electric field, in: Saratov Fall Meeting 2006: Laser Physics and Photonics, Spectroscopy and Molecular Modeling VII, eds. V.L. Derbov, L.A. Melnikov, and L.M. Babkov, Proc. SPIE 6537, article id. 653707 (2007), http:// dx.doi.org/10.1117/12.754004

[11]S.A. Smolyansky, M. Bonitz, and A.V. Tarakanov, Strong field generalization of the interband transitions kinetics, Phys. Part. Nucl. 47(7), 1075-1078 (2010).

[12] S.A. Smolyansky, A.V. Tarakanov, and M. Bonitz, Vacuum particle creation: analogy with the Bloch theory in solid state physics, Plasma Phys. 49(7-8), 575-584(2009), http://dx.doi.org/10.1002/ ctpp.200910058

[13] H. Bruus and K. Flensberg, Introduction to ManyBody Quantum Theory in Condensed Matter Physics (Copenhagen, 2002).

[14] O. Madelung, Introduction to Solid-State Theory, 1st ed. (Springer, Germany, 1978).

[15]F. Schwabl, Advanced Quantum Mechanics, ISBN10 3-540-25901-5, 3rd ed. (Springer, 2005).

[16]E. Klotins and G. Zvejnieks, Quantum chemical study of electron-phonon interaction in crystals, Phys. Status Solidi C 10(4), 705-708 (2013), http:// dx.doi.org/10.1002/pssc.201200872

[17] V. Pervushin, V. Skokov, A. Reichel, S. Smolyansky, and A. Prozorkevich, The kinetic description of vacuum particle creation in the oscillator representation, Int. J. Mod. Phys. A 20, 5689-5704 (2005).

[18]S.N. Data, Retarded boson-fermion interaction in atomic systems, J. Chem. Sci. 119(5), 351-356 (2007).

[19]J.C.R. Bloch, V.A. Mizerny, A.V. Prozorkevich, C.D. Roberts, S.M. Schmidt, S.A. Smolyansky, and D.V. Vinnik, Pair creation: Back reactions and damping, Phys. Rev. D 60, 116011 (1999), http:// dx.doi.org/10.1103/PhysRevD.60.116011

\title{
FONONŲ PALAIKOMA ELEKTRONŲ IR VAKANSIJŲ PORŲ SUSIDARYMO KINETIKA DVIEJŲ JUOSTŲ MODELYJE
}

\author{
E. Klotiņ̧̌ \\ Latvijos universiteto Kietojo kūno fizikos institutas, Ryga, Latvija
}

